\title{
Analysis on the Application Value of Full Seamless Service in Rehabilitation Area of Traditional Chinese Medicine
}

\author{
Chunxiao Ren ${ }^{1,}$, Guowu $\mathrm{Cao}^{2, \mathrm{~b}}$ \\ ${ }^{1}$ Shaanxi energy vocational and technical college, Xianyang, Shaanxi, 710200 \\ ${ }^{2}$ Xijing hospital, Xi'an, Shaanxi, 710032 \\ ${ }^{a}$ email, ${ }^{b}$ email
}

\begin{abstract}
Keywords: Full Seamless Service, Traditional Chinese Medicine Rehabilitation Area, Routine Care, Quality of Care
\end{abstract}

\begin{abstract}
Objective: To explore the application effect of full seamless service in rehabilitation area of traditional Chinese medicine. Methods: The patients were divided into two groups according to the standard of admission time in the rehabilitation area of traditional Chinese medicine in our hospital. They were given routine care (72 cases in control group) and seamless service (72 cases in observation group). The nursing effects of the two groups were evaluated. Results: The nursing quality scores of the nursing staff in the observation group were significantly higher than those in the control group $(\mathrm{P}<0.05)$. (94.4\%) was significantly higher than that of the control group (83.3\%) and significantly higher than that of the control group $(\mathrm{P}<0.05)$. Conclusion: The application of full seamless service in Chinese medicine rehabilitation area can effectively improve patient care satisfaction and improve the quality of nursing care, it is worthy of clinical popularization.
\end{abstract}

\section{Introduction}

Rehabilitation of traditional Chinese medicine is a very important type of clinical departments, to enhance the rehabilitation of patients, quality of life and so have a very important role [1]. This study will explore the full seamless service in Chinese medicine rehabilitation area of the application results are as follows:

\section{Materials and methods}

Clinical data. In this study, 144 patients were admitted to the time of Chinese medicine rehabilitation area in January 2015 -2016 February, with its admission time has been divided into two groups. There were 45 males and 27 females aged from 30 to 80 years (mean $56.6 \pm 6.7$ years) in the observation group (72 cases). There were 44 males and 28 females in the control group (72 cases) Year, mean (56.2 \pm 6.5$)$ years. There was no significant difference between the observation group and the control group in the statistical data $(\mathrm{P}>0.05)$.

Care methods. The patients in the control group were treated with routine nursing care, and the patients were given symptom introduction, symptom nursing, diet instruction and rehabilitation exercise.

Observation group of patients with seamless full application of quality services, care measures are as follows:

(1) seamless process care services: requiring nurses in the hospital patient until the discharge of the entire rehabilitation process must be in the patients need to immediately appear in front of patients, to provide the current process of care services. For example, when the patient is hospitalized, the caregiver should help to meet and inquire about the specific symptom and life content of the patient. The patient should be accompanied by the nursing staff to assist the patient and guide the patient to complete the examination. When the patient goes through the hospitalization procedure, he or she needs to ask the patient In the patient's daily care should also be asked in the daily care of patients on the quality of care of the current care of patients in the care of the patient's current quality of care needs, attention to the patient, , Care content views, ask patients 
on this hospital-related care content advice, the patient should be discharged by the nursing staff to explain the patient's condition and to explain the life of the relevant notes and referral content, the patient home After the need to have a home visit on a regular basis with the nursing staff and the actual situation for patients to explain their follow-up life should be corrected in the habit of content. In general, nurses in patients throughout the hospital stay should be in accordance with the patient hospital process to provide a comprehensive, continuous, seamless quality services;

(2) seamless staff care services: nursing staff for the families of patients should provide appropriate services to accompany patients in their families to actively improve the environment to the patient's family to actively explain the current clinical development of patients with the current situation, for the more anxious patient's family members But also should be given appropriate nursing care measures for patients with active family members of nursing staff can also be invited to the patient's family members to participate in the care of the patient content, combined with the patient's family to better understand the patient's patient care details, and For patients and their families to provide quality services related to the process.

Observation index [2]. (1)The quality of nursing staff was assessed by two groups of patients using the self-made quality of care questionnaire, which was mainly evaluated on the health education, nursing standards, nursing process and nursing attitude of nursing staff. The score range was 0-100 Points, the higher the score on behalf of patients with higher quality of care evaluation;

(2) The self - made nursing satisfaction questionnaire was used to evaluate the satisfaction of the nursing staff, and they were divided into three choices: satisfaction, satisfaction and dissatisfaction.

Statistical analysis. In this study, SPSS19.0 software was used to perform statistical analysis. The t-test was used to measure the data using $( \pm \mathrm{s})$ and the $\mathrm{x} 2$ test was used for the count data (\%). Test results show that $\mathrm{P}<0.05$, said there was a significant difference between the two, a statistically significant.

\section{Results}

Nursing quality assessment. There was significant difference between the observation group and the control group $(\mathrm{P}<0.05)$. See Table 1 .

Table 1 two groups of patients with nursing quality score comparison ( $\overline{\mathrm{x}} \pm \mathrm{s})$

\begin{tabular}{ccc}
\hline No. & Case & Nursing Quality Score \\
\hline Observation group & 72 & $89.2 \pm 8.6$ \\
Control group & 72 & $70.2 \pm 7.4$ \\
\hline
\end{tabular}

Care satisfaction assessment. (94.4\%) was significantly higher than that of the control group (83.3\%) and significantly higher than that of the control group $(\mathrm{P}<0.05)$. See Table 2.

Table 2 Comparison of two groups of patients with care satisfaction [n (\%)]

\begin{tabular}{cccccc}
\hline No. & Case & Very satisfied & Satisfied & Not satisfied & $\begin{array}{c}\text { Satisfaction } \\
(\%)\end{array}$ \\
\hline $\begin{array}{c}\text { Observation } \\
\text { group }\end{array}$ & 72 & 38 & 30 & 4 & 94.4 \\
Control group & 72 & 25 & 35 & 12 & 83.3 \\
\hline
\end{tabular}

\section{Conclusion}

Full seamless services are in the patient hospital discharge until the process by the nursing staff to provide patients with continuous whole process, comprehensive and in-depth quality care services. Quality of care throughout the process compared to the previous routine care for patients to provide comprehensive, continuous, in-depth quality care services, quality care model based on the further, standardizing the patient care services to the continuous process, so that patients throughout the hospital, discharged home and can enjoy the hospital during the meticulous care services. Clinical research results show that [3], full seamless service can help hospitals to reduce medical disputes and the incidence of medical malpractice, while improving the treatment of patients, prognosis and so have a positive help. In this study, the observation group in the nursing quality assessment and 
nursing satisfaction assessment results are better than the control group ( $\mathrm{P}<0.05)$, indicating the whole seamless service in the rehabilitation of the application of Chinese medicine can effectively improve patient care satisfaction And improve the quality of nursing care, it is worthy of clinical promotion.

\section{References}

[1] Yue Junyue. Quality care services in the application of Chinese medicine rehabilitation effect analysis [J]. Henan Medical Research, 2016, 25 (5): 957.

[2] Mo Yonglan. Full seamless service in the application of traditional Chinese medicine rehabilitation area [J]. Chinese Journal of Practical Nursing, 2011, 27 (29): 69-70.

[3] Liu Guorong, Liu Hongyan. Full seamless service in orthopedic diseases in hospital care [J]. Journal of Nursing, 2013, 19 (14): 105-106. 